\title{
Utilization of all components in biomass through high reducing sugar production and lignin nanoparticles preparation
}

Wei Liu

Tonghua Normal University

Shengnan Zhuo

Central South University

Xianfa Su

Henan Normal University

Mengying Si

Central South University

Kejing Zhang

Central South University

Aizi Tong

Tonghua Normal University

Yan Shi ( $\nabla$ shiyzyrs@csu.edu.cn )

Central South University https://orcid.org/0000-0001-6232-253X

Research

Keywords: corn straw, reducing sugar, THF-H2O pretreatment, lignin nanoparticles

Posted Date: December 20th, 2019

DOI: https://doi.org/10.21203/rs.2.19357/v1

License: (c) (i) This work is licensed under a Creative Commons Attribution 4.0 International License.

Read Full License 


\section{Abstract}

\section{Background}

Lignocellulosic biomass for biofuel production was considered as an effective way to develop new energy. However, the efficient sugar conversion of cellulose and practical utilization of lignin are great challenges for sustainable biorefinery. In addition, sugar conversion and lignin utilization are generally performed separately. In this study, high reducing sugar production and multiple lignin nanoparticles preparation were realized in a pattern based on tetrahydrofuran-water $\left(\mathrm{THF}-\mathrm{H}_{2} \mathrm{O}\right)$ pretreatment of corn straw (CS).

\section{Results}

The maximum production of the reducing sugar was $26.79 \mathrm{~g} / \mathrm{l}$, which was significantly higher than the theoretical yield of $20.65 \mathrm{~g} / \mathrm{l}$. Lignin nanoparticles with different sizes ranged from 239 to $798 \mathrm{~nm}$ were prepared using dissolved lignin in the supernatant fluid from different $\mathrm{THF}-\mathrm{H}_{2} \mathrm{O}$ pretreatment conditions through self-assembly with introducing water. The formation of lignin particles with different sizes were influenced by soluble lignin characteristics in the pretreatment liquid. The lignin molecular, functional groups, and structure were explored to elucidate the effects on the variation of lignin particles sizes by GPC, FTIR, and 2D-HSQC-NMR. The guaiacyl (G)-type lignin was easier to be dissolved in the mild pretreatment liquid, contributing to form smaller lignin nanoparticles with a good dispersibility. Comparatively, a small content of syringyl- and G-type lignin which caused by the lignin depolymerization retained in the severe pretreatment liquid to form the larger sphere particles.

\section{Conclusions}

The optimal pretreatment under $\mathrm{TH} 22\left(\mathrm{THF}-\mathrm{H}_{2} \mathrm{O}\right.$ pretreatment at $120^{\circ} \mathrm{C}$ for $2 \mathrm{~h}$ ) simultaneously realized the utilization of all components in biomass through high reducing sugar production and the smaller lignin particles preparation. This new pattern of CS pretreatment plays a novel perspective for the technical design of lignocellulosic biomass conversion.

\section{Background}

Due to the increasing consumption of energy and the depletion of fossil fuels, many researchers have dedicated to exploring the potential of alternative biofuels [1, 2]. Among different generations of biofuel development, lignocellulosic biomass is drawing increasing interest in producing fuels, chemicals, and materials $[3,4]$. Lignocellulose, consisting of cellulose, hemicelluloses and lignin, is the most abundant biomass on earth. For the decades, significant progress have been made to produce biofuels from lignocellulosic biomass [5, 6]. However, most of the biorefinery schemes focus on easily utilizing convertible celluloses and hemicelluloses, leaving lignin behind in the selective conversion processes [7, 8]. Lignin, together with cellulose and hemicellulose, constitutes a complex structure of lignocellulosic biomass with cross-linking heterogeneity. Therefore, how to efficiently utilize lignin with low cost and 
aromatic nature to realize all components utilization has turned into a global conundrum in current biorefinery.

There is no doubt that achieving high content of glucose is the major goal in the advanced biorefinery process. However, cellulose is inter-crossing with lignin which covers in the outside of biomass to hinder the enzymatic hydrolysis of biomass. Therefore, the removal of the outer lignin in lignocellulosic biomass has become the primary mission via pretreatment methods. Currently, a great many pretreatment strategies based on acid, alkali and organic solvent et al. were employed to efficiently remove or solve lignin from lignocellulose, which improve the glucose releasing [9-11]. Unfortunately, large amounts of lignin are wasted in the flushing way of pretreatment spent liquor.

Lignin is a natural aromatic compound with complex structure, high carbon content, abundant bonds, and functional groups. Therefore, it is a good precursor for the preparation of carbon-based materials including lignin fiber, activated carbon, carbon foam, and lignin nanomaterials [11, 12]. Recently, nanoparticles preparation with lignin has gained increasing interest, given their diverse advanced functionalities and great potential in the fields of medicine, catalysis, water treatment adsorbents, and antimicrobial agents [13]. It opens up a green and environment-friendly window for value-added utilization of lignocellulosic biomass. Various strategies have been developed for lignin nanoparticles preparation. However, most of the approaches have used lignins from the paper and pulping industry or biorefinery process, which need further chemical modification. For example, the uniform colloidal spheres were prepared via self-assembly with alkali lignin recovered from the pulping black liquor and chemically modified by acetylating [14]. Recently, lignin nanoparticles directly prepared with lignin produced from lignocellulose pretreatment were proposed $[15,16]$, which not only avoids lignin modification, but also realizes the utilization of waste lignin. However, the preparation of lignin nanoparticles and enzymatic hydrolysis of carbohydrates from lignocellulosic biomass are different and independent processes, therefore, the lignin with suitable structure for excellent colloidal spheres formation is usually obtained at the expense of sugar yield, due to the accessibility of carbohydrate to enzymes resulted from the destruction of lignin in the lignocellulosic biomass is not good enough.

In our previous studies, a new strategy involved a synergy of lignocelluloses pretreatment by THF- $\mathrm{H}_{2} \mathrm{O}$ and bacterial strain to enhance enzymatic hydrolysis was developed $[17,18]$. The pretreatment of CS with $\mathrm{THF}-\mathrm{H}_{2} \mathrm{O}$ alone had a high lignin removal efficiency and retained a large amount of cellulose for sugar production. However, the pretreatment spent liquor after THF recovery was directly treated as wastewater . In addition, lignin nanoparticles prepared based on alkali lignin dissolved in THF has been reported [14, 19]. Therefore, lignin nanoparticles directly prepared from the supernatant of CS pretreated by $\mathrm{THF}-\mathrm{H}_{2} \mathrm{O}$ to realize the utlization of lignin waste is reasonable and has great perspective for biorefinery design.

In this work, we developed a new pretreatment pattern based on acid-catalyzed $\mathrm{THF}-\mathrm{H}_{2} \mathrm{O}$ system, which not only obtained high reducing sugar yield from biomass, but also realized the untilization of lignin in wastewater through directly lignin nanopaticle preperation. Firstly, the acid-catalyzed THF-water pretreatment of CS was carried out. After enzymatic hydrolysis of the remaining solid residues, the yields 
of reducing sugar were investigated. In addition, lignin nanoparticles with different particle sizes were directly formed by self-assembly of the lignin in the pretreated supernatant under different conditions via adding water. The size, morphology, functional groups, and formation process of lignin nanoparticles were characterized. Then the structure changes of lignin in pretreatment solution were analyzed to explain the effect of the structure changes of lignin on the formation of lignin nanoparticles.

\section{Results And Discussion}

\subsection{Efficient biomass pretreatment}

\subsubsection{The release of reducing sugar}

Generally, biomass pretreatment is the primary step for the utilization of biomass resources. The satisfying enzymatic digestibility to produce sugar is the major criteria for measuring the effects of pretreatments. Enzymatic hydrolysis of CS under all pretreatment conditions was carried out and the corresponding reducing sugar yield was determined (Figure 1).

All the reducing sugar yields produced from CS that pretreated by $\mathrm{THF}-\mathrm{H}_{2} \mathrm{O}$ system under different conditions were higher than the theoretical reducing sugar yield of $20.65 \mathrm{~g} / \mathrm{l}$ (the value was calculated according to the literature [20]), except that of $10.19 \mathrm{~g} / \mathrm{I}$ from CS pretreated under $120^{\circ} \mathrm{C}$ for $1 \mathrm{~h}$ (TH21). The maximum reducing sugar yield of $26.79 \mathrm{~g} / \mathrm{l}$ was obtained after pretreatment under $150{ }^{\circ} \mathrm{C}$ for $2 \mathrm{~h}$ (TH52). The reducing sugar concentration higher than the theoretical has obtained in several previous studies with DNS methods, however, such a high yield of the reducing sugar in our work is of seldom (Table S1). In addition, the yield of the reducing sugar increased from $10.19 \mathrm{~g} / \mathrm{l}$ (TH21) to $26.28 \mathrm{~g} / \mathrm{l}(120$ ${ }^{\circ} \mathrm{C}$ for $4 \mathrm{~h}$, TH24) with the prolongation of reaction time under $120^{\circ} \mathrm{C}$. This is 3.74 and 11.40 -fold higher than that of the untreated sample $(84.70 \mathrm{mg} / \mathrm{g}$, that is $2.12 \mathrm{~g} / \mathrm{l}$ [17]). This phenomenon is attributed to the breakdown of the biomass recalcitrance structure. A large amount of lignin was dissolved by THF- $\mathrm{H}_{2} \mathrm{O}$, and the solid of cellulose residue species are accordingly exposed, which promoted the accessibility of the enzymes to the substrate. In general, the reducing sugar yield of biomass pretreated by $\mathrm{THF}-\mathrm{H}_{2} \mathrm{O}$ increases with the increase of the pretreated temperature and time [18]. It was surprised to observed that the reducing sugar yield of $\mathrm{CS}$ pretreated at $150^{\circ} \mathrm{C}$ increased from $24.26 \mathrm{~g} / \mathrm{l}\left(150{ }^{\circ} \mathrm{C}\right.$ for $\left.1 \mathrm{~h}, \mathrm{TH} 51\right)$ to $26.79 \mathrm{~g} / \mathrm{l}\left(150^{\circ} \mathrm{C}\right.$ for $\left.2 \mathrm{~h}, \mathrm{TH} 52\right)$ first, and then decreased to $25.47 \mathrm{~g} / \mathrm{l}\left(150^{\circ} \mathrm{C}\right.$ for $\left.4 \mathrm{~h}, \mathrm{TH} 54\right)$ with the increase of the pretreated time. Although enzymatic digestion was significantly improved due to lignin removal from $\mathrm{CS}$ by THF- $\mathrm{H}_{2} \mathrm{O}$ under $120^{\circ} \mathrm{C}$, there is still lignin remained in the pretreated $\mathrm{CS}$, which still has a negative effect on the enzymatic hydrolysis. This lignin residue can be further dissolved under more severe condition, which resulted that the reducing sugar yields under $150{ }^{\circ} \mathrm{C}$ are higher than those under $120^{\circ} \mathrm{C}$ with corresponding pretreated times. It is well known that lignin can provide the necessary attachment site for the enzymes, therefore, a small amount of lignin remains on the biomass is indispensable. However, almost all lignin and some of cellulose were removed from the CS, which lead to the decreased reducing sugar yield under TH54. 


\subsubsection{Morphology change of CS}

The effect of biomass pretreatment is closely related to the change of its structure. To deep understand the reducing sugar production from pretreated CS, we analyzed the morphology structure change of CS. The purpose of pretreatment is not only to remove lignin on the surface of biomass as much as possible but also to maximize the retention of cellulose to obtain a high yield of the reducing sugar.

The surface of the untreated CS (Figure 2a) is smooth and rigid, covered with a large amount of lignin, which is the physical barrier for enzymatic hydrolysis of biomass. In contrast, the surface of CS pretreated under TH21 was changed (Figure 2b). Some lignin droplets were deposited on the surface of the pretreated residues $₫$ which was similar to the phenomenon that was found in our previous work [9]. The formation of lignin droplets was caused by the acidic environment created by adding sulfuric acid as a catalyst in $\mathrm{THF}-\mathrm{H}_{2} \mathrm{O}$ reaction system. However, the lignin droplets remained on the surface of $\mathrm{CS}$ hindered the enzymatic hydrolysis, resulting in the low yield of the reducing sugar under TH21. In contrast, the morphology of CS pretreated under the other five reaction conditions has undergone tremendous changes. For example, the CS pretreated by $\mathrm{TH} 22$ was split and the cellulose fiber appeared (Figure 2c). The CS was deformed after TH24 pretreatment (Figure 2d), indicating that the structure of the CS became fragile and collapsed [21]; TH51 pretreated CS was also cracked, showing cellulose structure (Figure 2e). Moreover, the fiber bundles can be seen in TH52 pretreated CS (Figure 2f) $₫$ which significantly improves the accessibility of cellulose to the enzyme, contributing to the highest reducing sugar yield. With the aggravation of reaction conditions (TH54), the CS was damaged to form a slurry structure (Figure $2 \mathrm{~g}$ ) that was not good as that of TH52, causing the reducing sugar yield decrease.

\subsubsection{Mass loss of the CS}

In order to further investigate the change of the CS structure before and after pretreatment, the analysis of the quality loss of the CS under various conditions was performed. The mass loss of treated CS increases with the prolongation of reaction time at $120^{\circ} \mathrm{C}$ and $150{ }^{\circ} \mathrm{C}$ (Figure 3 ).

The CS used in this study was found to consist of $34.9 \%$ of cellulose, $38.5 \%$ of hemicellulose, $13.9 \%$ of lignin, and $12.7 \%$ of other non-organic and organic components [17]. The mass loss of CS under the reaction condition of $\mathrm{TH} 21$ was $36.14 \%$. Moreover, the reducing sugar yield was low and the morphology of the CS was not significant changed after pretreatment under this condition (Section 2.1.1 and 2.1.2). It can be inferred that the mass loss of CS is mainly attributed to the removal of a small amount of lignin and other components like hemicellulose. Most of the lignin formed droplets deposited on the surface of the CS. The mass losses of $\mathrm{TH} 22$ and $\mathrm{TH} 24$ were $50.5 \%$ and $52 \%$ respectively, which were contributed by most of the hemicellulose and lignin removal. Similarly, with the increase of reaction time, more lignin was removed and more mass loss was observed at $150^{\circ} \mathrm{C}$. In addition, under TH54 condition, some cellulose was destroyed after lignin removal, which resulted in the increase of reducing sugar yield first and then decrease. Therefore, in order to maximize the yield of the reducing sugar from biomass, it is very important to select appropriate pretreatment conditions. Compared with the other THF pretreatment [22, 
23], the pretreatment method adopted in this paper has the advantages of low temperature, low energy consumption, and high sugar yield. It is an environmentally friendly pretreatment technology.

\subsection{Formation of lignin nanoparticles}

The current utilization of biomass resources is not only involved in the utilization of cellulose components, but also in lignin due to its unique structure. Therefore, in addition to the high reducing sugar obtained from $\mathrm{CS}$ by $\mathrm{THF}-\mathrm{H}_{2} \mathrm{O}$ pretreatment, we also directly used the pretreatment liquid to prepare lignin nanoparticles via introducing water into it without other chemical modification, which made the utilization of lignin in biomass. Different concentrations of colloid solution were formed under six different reaction conditions (Figure S1). To understand the characteristics of the colloid solution, we adopted SEM to observe the morphology of lignin nanoparticles, nano size analyzer to get information of particle size distribution statistics, Uv-vis to measure the concentration of colloid solution, and FTIR to analyze the functional groups of lignin particles.

\subsubsection{Morphology and Size analysis of lignin nanoparticles}

All the prepared lignin nanoparticles are spherical and rich (Figure 4 and Table S2). The particle size increases with the increase of the reaction time under the temperatures of $120^{\circ} \mathrm{C}$ and $150{ }^{\circ} \mathrm{C}$. Lignin nanoparticles prepared from TH21 pretreatment solution (NP-TH21) has a small number of very small nanoparticles, and the particle size mainly distributes between $164-295 \mathrm{~nm}$, the average particle size is $239 \mathrm{~nm}$. The size distribution of lignin nanoparticles prepared from TH22 pretreatment solution (NPTH22) was 164-342 nm, which was a little bigger than that of NP-TH21. Its average particle size is 243 $\mathrm{nm}$ and the dispersion of particles is better than that of NP-TH21. Lignin nanoparticles prepared from TH24 pretreatment solution (NP-TH24) has a particle size distribution of 295-531 nm with the average particle size of $433 \mathrm{~nm}$. Besides, it can be observed that the minimum particle size of NP-TH24 is larger than the average particle size of NP-TH21 and NP-TH22. Moreover, the particle sizes of NP-TH51, NPTH52, and NP-TH54 prepared from TH51, TH52 and TH54 pretreatment solutions are also increasing. The corresponding particle sizes are 190-342, 459-712, 615-955 nm with the average particle sizes of 359, 589 and $798 \mathrm{~nm}$, respectively. These results indicated that the lignin particle size gradually grew with the increase of reaction intensity. In addition, some NP-TH54 particles even reach about 1000 nm. The explanation for this result might be related to the lignin structure dissolved in different pretreatment solution. In addition, the lignin nanopaticles prepared in this work was comparative to the other lignin nanoparticles with various shapes and sizes prepared by different methods (Table S3).

\subsubsection{Uv-vis analysis of lignin nanoparticles}

Uv-vis was used to compare the lignin in the pretreatment solution with the corresponding lignin nanoparticles to analyze the formation of lignin nanoparticles. Previous studies have discussed the lignin nanoparticles are formed by the $\pi-\pi$ interaction of orbitals of non-chemical bonds between aromatic rings in lignin molecule [14]. 
As displayed in Figure 5, the absorption peak of typical benzene compounds ( $280 \mathrm{~nm}$ ) occurred in the lignin and lignin nanoparticles solution, suggesting the aromatic ring structure of lignin has not destroyed in the process of lignin nanoparticles formation. It was consistent with the conclusion that $\pi-\pi$ interaction in lignin caused the hydrophobic effect which brought about the lignin colloid solution formation [14]. The different shifts of maximum I at $\sim 280 \mathrm{~nm}$ indicated the weakening or strengthening in $\pi$-stacking of aromatic groups. Moreover, the TH24, TH52, and TH54 of lignin-NP colloid solution had a higher absorption peak at maximum I, which were related to their high concentration that can be seen in the Figure S1. The higher concentration depends on the increasing lignin dissolution with the increase of pretreatment reaction degree.

\subsubsection{FTIR analysis of lignin nanoparticles}

The structural properties of materials determine their application values. Therefore, FTIR was used to characterize the functional groups of lignin nanoparticles. As shown in Figure 6, the abundant functional groups were intuitively observed in the lignin nanoparticles prepared from pretreatment solutions at 120 ${ }^{\circ} \mathrm{C}$ and $150^{\circ} \mathrm{C}$.

The broad absorption peak at $\sim 3400 \mathrm{~cm}^{-1}$ and the band at $\sim 1379 \mathrm{~cm}^{-1}$ represent $-\mathrm{OH}$ stretching of phenolic and aliphatic moieties. The band at $2918 \mathrm{~cm}^{-1}$ and $2849 \mathrm{~cm}^{-1}$ represents $\mathrm{C}-\mathrm{H}$ stretching [24]. Aromatic skeleton stretching occurs at $\sim 1600 \mathrm{~cm}^{-1}, \sim 1515 \mathrm{~cm}^{-1}, \sim 1426 \mathrm{~cm}^{-1}$, and $\mathrm{C}=0$ stretching in $\mathrm{G}$ unit appeared at $\sim 1267 \mathrm{~cm}^{-1}$ [25]. All these location peaks suggested that there is no significant change in the aromatic structures of lignin in the formation process of lignin nanoparticles; and the particle surface was modified with higher phenolic hydroxyl and carboxyl functional groups, which is good for enhancing the solubility and stability of lignin nanoparticles. In addition, lignin nanoparticles have extra function groups of $\mathrm{C}=0$ (at $\sim 1330 \mathrm{~cm}^{-1}$ and $\sim 1265 \mathrm{~cm}^{-1}$ ) and C-O-C (at $\sim 1165 \mathrm{~cm}^{-1}$ ) compared to the lignin. In recent years, the lignin nanoparticles with plenty of functional groups have been reported to play an important role in the adsorption materials applied in the environment field and other composite materials preparation for biomedical or cosmetic fields $[12,26]$. Therefore, the lignin nanoparticles in our study have the potential to be applied in these fields.

\subsection{Influence of lignin on the lignin nanoparticles}

Spherical lignin nanoparticles with different sizes were prepared in different pretreatment solution. The formation of lignin nanoparticles is closely related to the structure of dissolved lignin [27], therefore, we extracted the lignin from the liquid and analyzed their molecular weight and structural changes by GPC, FTIR, and 2D-HSQC-NMR to elucidate the impact mechanism of lignin structure on the formation of lignin nanoparticles.

\subsubsection{GPC analysis}

The analysis of the changes in lignin molecular weight through GPC can be used to characterize the degree of lignin polymerization during $\mathrm{THF}-\mathrm{H}_{2} \mathrm{O}$ pretreatment. The deconstruction of the macromolecular 
lignin during the pretreatment process can be monitored via three indexes including weight-average molecular weight $\left(M_{w}\right)$, number-average molecular weight $\left(M_{n}\right)$, and dispersive poly-dispersity (PDI).

Lignin extracted from different pretreatment liquids showed similar $M_{w}$ distribution ranging from $2670 \mathrm{Da}$ to $3644 \mathrm{Da}$ (Table 1). Compared with the untreated lignin (9742 Da), the $\mathrm{M}_{\mathrm{w}}$ of pretreated CS was significantly reduced, indicating that $\mathrm{THF}-\mathrm{H}_{2} \mathrm{O}$ solvent depolymerized the macromolecular lignin in the pretreatment process [28]. Most of the lignin (in the form of polymer) after depolymerization was dissolved in the pretreatment solution. Meanwhile, the $\mathrm{M}_{\mathrm{w}}$ of lignin in the pretreatment solution gradually increased when the pretreatment conditions changed from TH21 to TH51, indicating that more macromolecular lignin was dissolved in $\mathrm{THF}-\mathrm{H}_{2} \mathrm{O}$ solvent with the increase in severity of the reactions.. The results are consistent with the particle size changes of lignin nanoparticles obtained in the above. In addition, the $\mathrm{M}_{\mathrm{w}}$ of lignin from TH54 and TH52 did not continue to increase, which may be attributed to the strong fragmentation of lignin caused by excessive pretreatment, followed by different degrees of the condensation reaction of small molecular lignin. The PDI analysis showed that molecular weight distribution under the weakest condition (TH21) was the narrowest and the corresponding $\mathrm{M}_{w}$ was the smallest. The increase in severity of the reactions resulted in the larger molecular weight, wider distribution, and worse homogeneity of lignin. The PDI of TH52 and TH54 is the same as that of TH22, but their $\mathrm{M}_{\mathrm{w}}$ are lower than that of TH22. In addition, the size of NP-TH52 and NP-TH54 are much larger than those of NP-TH22. This phenomenon may be attributed to the different types of structural units of lignin. Therefore, lignin was further characterized by FTIR and 2D-HSQC-NMR analysis to explain it.

\subsubsection{FTIR analysis}

Based on the results of reducing sugar yield and the characteristics of the prepared lignin nanoparticles, the lignins under the condition of TH22 and TH52 were selected for FTIR and 2D- HSQC-NMR analysis to further investigate the effect of lignin structure on the size of nanoparticles.

Obviously, FTIR spectra ( Figure 7) shows that most of the characteristic peaks of lignin extracted from TH22 and TH52 pretreatment liquids still remained in these two samples [29]. The peaks at $1617 \mathrm{~cm}^{-1}$, $1513 \mathrm{~cm}^{-1}$, and $1426 \mathrm{~cm}^{-1}$ are related to the vibration of aromatic ring skeleton, and the peak at $1462 \mathrm{~cm}^{-1}$ represents the $\mathrm{C}-\mathrm{H}$ deformation of aromatic ring skeleton. These peaks indicated the existence of benzene structure in lignin of TH22 and TH52. However, compared with lignin of TH22, the peak at 1320 $\mathrm{cm}^{-1}$ which represents $\mathrm{C}=0$ stretching in syringyl (S) derivatives disappeared in lignin of TH52, while it still retained a little in lignin of $\mathrm{TH} 22$. In addition, the $\mathrm{G}$ ring breathing with $\mathrm{C}=0$ stretching $\left(\right.$ at $\left.1270 \mathrm{~cm}^{-1}\right)$ displays significantly decrease from TH52 lignin. Moreover, the peak at $1164 \mathrm{~cm}^{-1}$ which is attributed to the C-O-C vibration only remained in TH22 lignin. The peak at $1227 \mathrm{~cm}^{-1}$ associated with $\mathrm{C}-\mathrm{C}, \mathrm{C}-\mathrm{O}$, and $\mathrm{C}=\mathrm{O}$ stretching only appeared in TH52 lignin. These changes may be caused by the breakage of linkage bonds due to the intense pretreatment. The destruction of linkages resulted in the changes of lignin structure units such as S and G. In order to further confirm the speculation, we adopted the 2D-HSQCNMR analysis to illustrate the structural changes of lignin. 


\subsubsection{D-HSQC-NMR analysis}

Generally, the lignin is consisted of three basic units $(\mathrm{S}, \mathrm{G}$ and p-hydroxyphenyl $(\mathrm{H}))$ and linked by $\beta-0-4$, $\beta-\beta, \beta-5$ etc. [30]. All these signals can be reflected through the 2D-NMR spectra. The signal information of side chain region $\left(\delta_{C} / \delta_{H} 50-90 / 2.5-6.0\right)$ and aromatic region $\left(\delta_{C} / \delta_{H} 100-135 / 5.5-8.5\right)$ of lignin extracted from TH22 and TH52 pretreatments was displayed in Figure 8. And the main cross-signals of lignin related to HSQC spectra were also listed in Table S4.

The signals of $\beta-0-4$ and $\beta-5$ bonds in lignin-TH22 is stronger than that of lignin-TH52, indicating the lignin in the dissolved process was destroyed severely under TH52. In the aromatic region, the S-type and G-type lignin in TH52 pretreatment solution significantly decreased, and even the signal of H-type lignin completely disappeared. In contrast, the signals of all three basic lignin units (S, G and H-type) remained in TH22 pretreatment solution. Specifically, the G-type lignin signal in TH22 lignin is much higher than that of TH52 lignin. This phenomenon may attribute to the lignin recombination and condensation that happened in the acidic environment under mild conditions (TH22) after the depolymerization [31]. It is worth noting that it is easier for high content of G-type lignin or low S/G lignin to form small size nanoparticles [15]. Therefore, in order to achieve the preparation of lignin nanoparticles with smaller particle size, the reaction solvent must have the ability to dissolve more G-type lignin. Correspondingly, the smaller nanoparticles prepared from TH22 than those fromTH52 in our work can be explained.

The pretreatment under TH52 can not form good lignin nanoparticles even if the maximum yield of reducing sugar can be produced. However, pretreatment under TH22, which also exceeds the theoretical yield of the reducing sugar, can simultaneously realize the preparation of lignin nanoparticles with good properties and high reducing sugar yield. Therefore, the pretreatment under $\mathrm{TH} 22$ was more practical in the actual biorefinery. The schematic process flow diagram was fabricated as shown in Figure 9 . This strategy provides a practical guiding significance for the design of biomass pretreatment and the preparation of lignin nanoparticles.

\section{Conclusions}

An efficient pretreatment strategy based on $\mathrm{THF}-\mathrm{H}_{2} \mathrm{O}$ with the acid catalytic system is proposed in this paper. Under the optimum pretreatment condition $\left(120^{\circ} \mathrm{C}, 2 \mathrm{~h}\right)$, the reducing sugar yield of $24.23 \mathrm{~g} / \mathrm{I}$ was higher than the theoretical value, and lignin nanoparticles with good dispersibility and particle size of 243 $\mathrm{nm}$ were obtained. The mechanism of simultaneously obtaining the high reducing sugar yield and nanoparticles with small size in biomass pretreatment was elucidated. $\mathrm{THF}-\mathrm{H}_{2} \mathrm{O}$ pretreatment that dissolved more G-type lignin would achieve better enzymatic hydrolysis efficiency and smaller lignin nanoparticles. This pretreatment strategy realizes the effective utilization of all components in biomass.

\section{Materials And Methods}

\subsection{Materials and Chemicals}


The corn straw (CS) was purchased from Jining, Shandong province, China. The CS after washing and drying was cut and crushed using a pulverizer, then sifted by 18 to 60-mesh griddles and air-dried for $24 \mathrm{~h}$ at $50^{\circ} \mathrm{C}$ before use. Tetrahydrofuran (THF, A.R.) was purchased from a commercial Shanghai Macklin Biochemical Co., Ltd. Concentrated sulfuric acid (98 wt\%) was purchased from ChengDu Chron Chemicals Co., Ltd. Other chemicals were purchased from a commercial source and used without any further purification.

\subsection{Biomass pretreatment}

The CS was weighed at the ration of $1: 20(\mathrm{~g} / \mathrm{ml})$ with the THF/ $\mathrm{H}_{2} \mathrm{O}(1: 1)$ solution. The mixture was placed in a $100 \mathrm{ml}$ of hydrothermal reactor. Then the sulfuric acid $(0.5 \mathrm{wt} \%)$ was added as the catalyst. The hydrothermal reactor was put into the air drying oven after stirring. The temperature and time were set to $120^{\circ} \mathrm{C}$ and $150^{\circ} \mathrm{C}$ for $1 \mathrm{~h}, 2 \mathrm{~h}$, and $4 \mathrm{~h}$, respectively. The reactor was quickly cooled down by flushing the water after the completion of the reaction. Then, the solid residues and reaction liquid were separated by vacuum filtration. The liquid was collected and stored in a refrigerator $\left(4^{\circ} \mathrm{C}\right)$. The solid residues were further filtrated by distilled water to remove THF until the $\mathrm{pH}$ was neutral. And then, they were dried at $50^{\circ} \mathrm{C}$ for $24 \mathrm{~h}$.

\subsubsection{Enzymatic hydrolysis}

The enzymatic hydrolysis of air-dried untreated and pretreated solid residues was conducted with Cellulase Cellic (CTec2, Novozymes) in the water bath shaker at $50^{\circ} \mathrm{C}, 150 \mathrm{rpm}$ for $3 \mathrm{~d}$. The supernatant with $100 \mu \mathrm{L}$ was withdrawn out to measure the reducing sugar with the DNS method [32]. All the experiments were done in triplicate.

\subsubsection{Weight loss analysis}

The weight loss rate of CS was calculated with the different mass obtained before and after pretreatment [33]. All the experiments were done in triplicate.

\subsubsection{Morphology analysis}

The morphology of CS before and after pretreatments with different conditions were detected with the scanning electron microscopy (SEM JSM-IT300LA, JEOL, Japan) [34].

\subsection{Preparation of Lignin nanoparticles}

Lignin stream from the biomass pretreatment under different conditions was transformed into a flask. Subsequently, the excess of the deionized water was slowly added into each flask with constant magnetic stirring. Finally, the colloid solutions containing lignin nanoparticles from different pretreatment conditions were obtained as shown in Figure S1.

\subsection{Characterization of lignin nanoparticles}


The morphology of lignin nanoparticles was observed with the field emission scanning electron microscope (FESEM, FEI Nova NanoSEM230, Czekh). The size and dispersity of particles were analyzed using a Nano-ZS (Malvern Instruments, UK). The absorption peak of lignin nanoparticles in aqueous solutions was measured using a U-4100 Spectrophotometer (Thermo Scientific Inc., USA). The purified lignin directly extracted from the pretreatment liquid was used as the positive control. The extracted lignin was dissolved in the solution of THF and water at the ratio of 1:1. The chemical groups of lignin nanoparticles were confirmed via a Nicolet IS10 Fourier Transform Infrared spectrometer (FTIR, Thermo Fisher, USA).

\subsection{Extraction and Characterization of lignin}

In order to study the influence of lignin structure on the formation of lignin nanoparticles, the lignin in the pretreatment liquid was extracted with the same method as the previous report [35]. The extracted lignin samples were analyzed via a Bruker Avance $400 \mathrm{MHz}$ two-dimensional heteronuclear single quantum coherence nuclear magnetic resonance spectrometer (2D- HSQC-NMR, Billerica, MA, USA) to identify the change of lignin structure units and investigate the cleavage of linkage bonds. The chemical groups of lignin were analyzed through the FTIR. The Waters Alliance gel permeation chromatography 1515 (Waters, USA) was used to analyze the molecular weight of lignin.

\section{Abbreviations}

\begin{tabular}{cc}
\hline Abbreviation & Full name \\
\hline CS & Corn straw \\
THF-H ${ }_{2} \mathrm{O}$ & Tetrahydrofuran-water \\
G & Guaiacyl \\
S & Syringyl \\
TH21 & $120{ }^{\circ} \mathrm{C}$ for $1 \mathrm{~h}$ \\
TH22 & $120{ }^{\circ} \mathrm{C}$ for $2 \mathrm{~h}$ \\
TH24 & $120{ }^{\circ} \mathrm{C}$ for $4 \mathrm{~h}$ \\
TH51 & $150{ }^{\circ} \mathrm{C}$ for $1 \mathrm{~h}$ \\
TH52 & $150{ }^{\circ} \mathrm{C}$ for $2 \mathrm{~h}$ \\
TH54 & $150{ }^{\circ} \mathrm{C}$ for $4 \mathrm{~h}$ \\
NP-THxx & poly-dispersity \\
PDI & nanoparticles prepared from THxx pretreatment \\
Mw & weight-average molecular weight \\
Mn & number-average molecular weight \\
FESEM & Field emission scanning electron microscope \\
FTIR & Fourier Transform Infrared spectrometer \\
Uv-vis & Ultraviolet visible spectroscopy \\
GPC & Gel permeation chromatography \\
HSQC-NMR & Heteronuclear single quantum coherence nuclear magnetic resonance \\
\hline
\end{tabular}




\section{Declarations}

\section{Ethics approval and consent to participate:}

Not applicable.

\section{Consent for publication:}

Not applicable.

\section{Availability of data and material:}

The datasets used and/or analyzed during the current study are available from the corresponding author on reasonable request.

\section{Competing interests:}

The authors declare that they have no competing interests.

\section{Authors' contributions}

WL designed, guided, and supported the experiment, and participated in revising. SNZ performed the experiments and analyzed the data, drew the figures and tables, wrote the draft. XFS, MYS, KJZ and AZT co-analyzed the results, read and provided the suggestion to the manuscript. YS contributed to the design, writing, and revising of the manuscript. All authors read and approved the final manuscript.

\section{Acknowledgments}

The authors thank the Chinese National Engineering Research Center for Control \& Treatment of Heavy Metal Pollution for proving the instruments tests.

\section{Funding}

This work was supported from the Program of Jilin Provincial Education Department (TJKH20170438KJ) and Key R\&D Program of Hunan Province (2018SK2044).

\section{References}

1. Huber SI, Avelino C. Synthesis of transportation fuels from biomass chemistry, catalysts, and engineering. ChemRev. 2006;106:4044-4098.

2. Silva EABd, Zabkova M, Araújo JD, Cateto CA, Barreiro MF, Belgacem MN, Rodrigues AE. An integrated process to produce vanillin and lignin-based polyurethanes from Kraft lignin. Chem Eng Res Des. 2009;87:1276-1292. 
3. Pandey MP, Kim CS. Lignin Depolymerization and Conversion: A Review of Thermochemical Methods. Chem Eng Technol. 2011;34:29-41.

4. Sturgeon MR, Kim S, Lawrence K, Paton RS, Chmely SC, Nimlos M, Foust TD, Beckham GT. A Mechanistic Investigation of Acid-Catalyzed Cleavage of Aryl-Ether Linkages: Implications for Lignin Depolymerization in Acidic Environments. ACS Sustain Chem Eng. 2013;2:472-485.

5. Himmel ME, Ding SY, Johnson DK, Adney WS, Nimlos MR, Brady JW, Foust TD. Biomass recalcitrance: engineering plants and enzymes for biofuels production. Science. 2007;315:804-807.

6. Peralta-Yahya PP, Zhang F, del Cardayre SB, Keasling JD. Microbial engineering for the production of advanced biofuels. Nature. 2012;488:320-328.

7. Chundawat SP, Beckham GT, Himmel ME, Dale BE. Deconstruction of lignocellulosic biomass to fuels and chemicals. Annu Rev Chem Biomol Eng. 2011;2:121-145.

8. Ragauskas AJ, Beckham GT, Biddy MJ, Chandra R, Chen F, Davis MF, Davison BH, Dixon RA, Gilna P, Keller M. Lignin valorization: improving lignin processing in the biorefinery. Science. 2014;344:1246843.

9. Yan X, Wang Z, Zhang K, Si M, Liu M, Chai L, Liu X, Shi Y. Bacteria-enhanced dilute acid pretreatment of lignocellulosic biomass. Bioresour Technol. 2017;245:419-425.

10. Liu D, Yan X, Zhuo S, Si M, Liu M, Wang S, Ren L, Chai L, Shi Y. Pandoraea sp. B-6 assists the deep eutectic solvent pretreatment of rice straw via promoting lignin depolymerization. Bioresour Technol. 2018;257:62-68.

11. Si M, Zhang J, He Y, Yang Z, Yan X, Liu M, Zhuo S, Wang S, Min X, Gao C. Synchronous and rapid preparation of lignin nanoparticles and carbon quantum dots from natural lignocellulose. Green Chem. 2018;20:3414-3419.

12. Duval A, Lawoko M. A review on lignin-based polymeric, micro- and nano-structured materials. React Funct Polym. 2014;85:78-96.

13. Nair SS, Sharma S, Pu Y, Sun Q, Pan S, Zhu JY, Deng Y, Ragauskas AJ. High Shear Homogenization of Lignin to Nanolignin and Thermal Stability of Nanolignin-Polyvinyl Alcohol Blends. ChemSusChem. 2014;7:3513-3520.

14. Qian Y, Deng Y, Qiu X, Li H, Yang D. Formation of uniform colloidal spheres from lignin, a renewable resource recovered from pulping spent liquor. Green Chem. 2014;16:2156.

15. Liu Z, Hao N, Shinde S, Pu Y, Kang X, Ragauskas AJ, Yuan J. Defining lignin nanoparticle properties through tailored lignin reactivity by sequential organosolv fragmentation approach (SOFA). Green Chem. 2019;21:245-260.

16. Tian D, Hu J, Bao J, Chandra RP, Saddler JN, Lu C. Lignin valorization: lignin nanoparticles as highvalue bio-additive for multifunctional nanocomposites. Biotechnol Biofuels. 2017;10:192.

17. Zhuo S, Yan X, Liu D, Si M, Zhang K, Liu M, Peng B, Shi Y. Use of bacteria for improving the lignocellulose biorefinery process: importance of pre-erosion. Biotechnol Biofuels. 2018; 11:146. 
18. Zhuo S, Peng B, Yan X, Zhang K, Si M, Liu M, Shi Y. Conquering lignin recalcitrance by Pandoraea sp. B-6 to improve co-solvent pretreatment of corn stover. Process Biochem. 2018; 75:187-193.

19. Lievonen M, Valle-Delgado JJ, Mattinen M-L, Hult E-L, Lintinen K, Kostiainen MA, Paananen A, Szilvay GR, Setälä H, Österberg M. A simple process for lignin nanoparticle preparation. Green Chem. 2016;18:1416-1422.

20. Wang W, Yuan T, Wang K, Cui B, Dai Y. Combination of biological pretreatment with liquid hot water pretreatment to enhance enzymatic hydrolysis of Populus tomentosa. Bioresour Technol. 2012;107:282-286.

21. Cai CM, Zhang T, Kumar R, Wyman CE: THF co-solvent enhances hydrocarbon fuel precursor yields from lignocellulosic biomass. Green Chem. 2013;15:3140.

22. Cai CM, Nagane Nikhil , Kumar Rajeev , Wyman CE: Coupling metal halides with a co-solvent to produce furfural and 5-HMF at high yields directly from lignocellulosic biomass as an integrated biofuels strategy. Green Chem. 2014;16:3819-3829.

23. Jiang Z, He T, Li J, Hu C. Selective conversion of lignin in corncob residue to monophenols with high yield and selectivity. Green Chem. 2014;16:4257-4265.

24. Mou H, Li B, Fardim P. Pretreatment of Corn Stover with the Modified Hydrotropic Method To Enhance Enzymatic Hydrolysis. Energ Fuel. 2014;28:4288-4293.

25. Tang C, Shan J, Chen Y, Zhong L, Shen T, Zhu C, Ying H. Organic amine catalytic organosolv pretreatment of corn stover for enzymatic saccharification and high-quality lignin. Bioresour Technol. 2017;232:222-228.

26. Demirbaş A. Adsorption of $\operatorname{Cr}(\mathrm{III})$ and $\operatorname{Cr}(\mathrm{VI})$ Ions from Aqueous Solutions on to Modified Lignin. Energy Sources 2005, 27:1449-1455.

27. Zhao W, Simmons B, Singh S, Ragauskas A, Cheng G. From lignin association to nano-/microparticle preparation: extracting higher value of lignin. Green Chem. 2016;18:5693-5700.

28. Yuan T, Sun S, Xu F, Sun R. Isolation and physico-chemical characterization of lignins from ultrasound irradiated fast-growing poplar wood. Bioresour. 2011;6:414-433.

29. Li S, Li W, Zhang Q, Shu R, Wang H, Xin H, Ma L. Lignin-first depolymerization of native corn stover with an unsupported MoS2 catalyst. RSC Advances. 2018;8:1361-1370.

30. Wen J, Sun S, Yuan T, Sun R. Structural elucidation of whole lignin from Eucalyptus based on preswelling and enzymatic hydrolysis. Green Chem. 2015;17:1589-1596.

31. Wen J, Yuan T, Sun S, Xu F, Sun R. Understanding the chemical transformations of lignin during ionic liquid pretreatment. Green Chem 2014;16:181-190.

32. Si M, Yan X, Liu M, Shi M, Wang Z, Wang S, Zhang J, Gao C, Chai L, Shi Y. In Situ Lignin Bioconversion Promotes Complete Carbohydrate Conversion of Rice Straw by Cupriavidus basilensis B-8. ACS Sustain Chem Eng. 2018;6:7969-7978.

33. Nan Y, Jia L, Yang M, Xin D, Qin Y, Zhang J. Simplified sodium chlorite pretreatment for carbohydrates retention and efficient enzymatic saccharification of silvergrass. Bioresour Technol. 
2018;261:223-231.

34. Zhang K, Si M, Liu D, Zhuo S, Liu M, Liu H, Yan X, Shi Y. A bionic system with Fenton reaction and bacteria as a model for bioprocessing lignocellulosic biomass. Biotechnol Biofuels. 2018;11:31.

35. Chai L, Liu M, Yan X, Cheng X, Zhang T, Si M, Min X, Shi Y. Elucidating the Interactive Impacts of Substrate-Related Properties on Lignocellulosic Biomass Digestibility: A Sequential Analysis. ACS Sustain Chem Eng. 2018;6:6783-6791.

\section{Table}

Table 1 Molecular weight of the lignin extracted from different pretreatment liquid

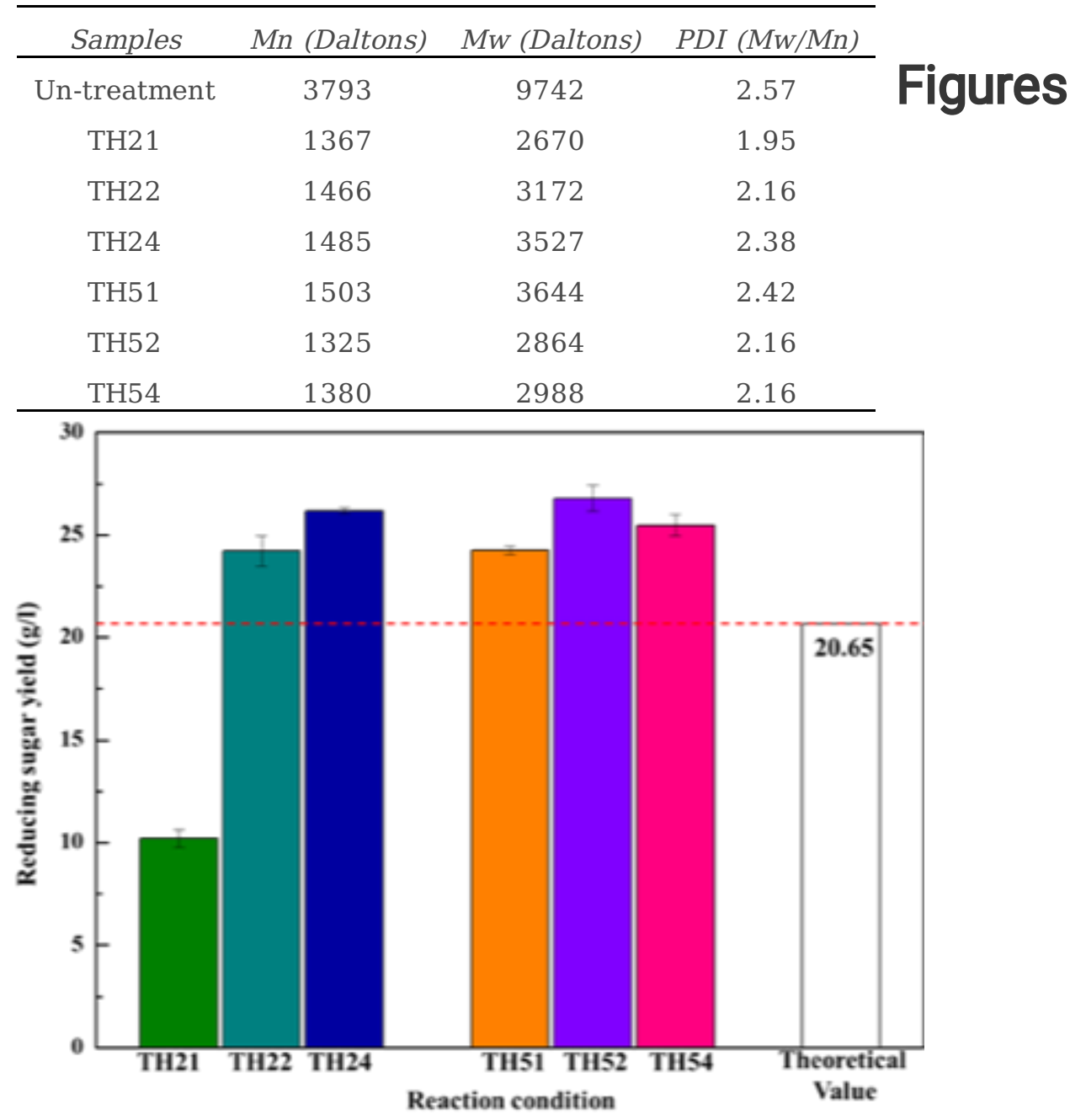

\section{Figure 1}

Reducing sugar yield of corn stover pretreatment under different conditions. THXX: T represents temperature, $\mathrm{H}$ represents the hour, $\mathrm{X}(2 / 5)$ and $\mathrm{X}(1 / 2 / 4)$ represent the temperature of $120{ }^{\circ} \mathrm{C} / 150{ }^{\circ} \mathrm{C}$ and the hour of $1 \mathrm{~h} / 2 \mathrm{~h} / 4 \mathrm{~h}$, respectively. 


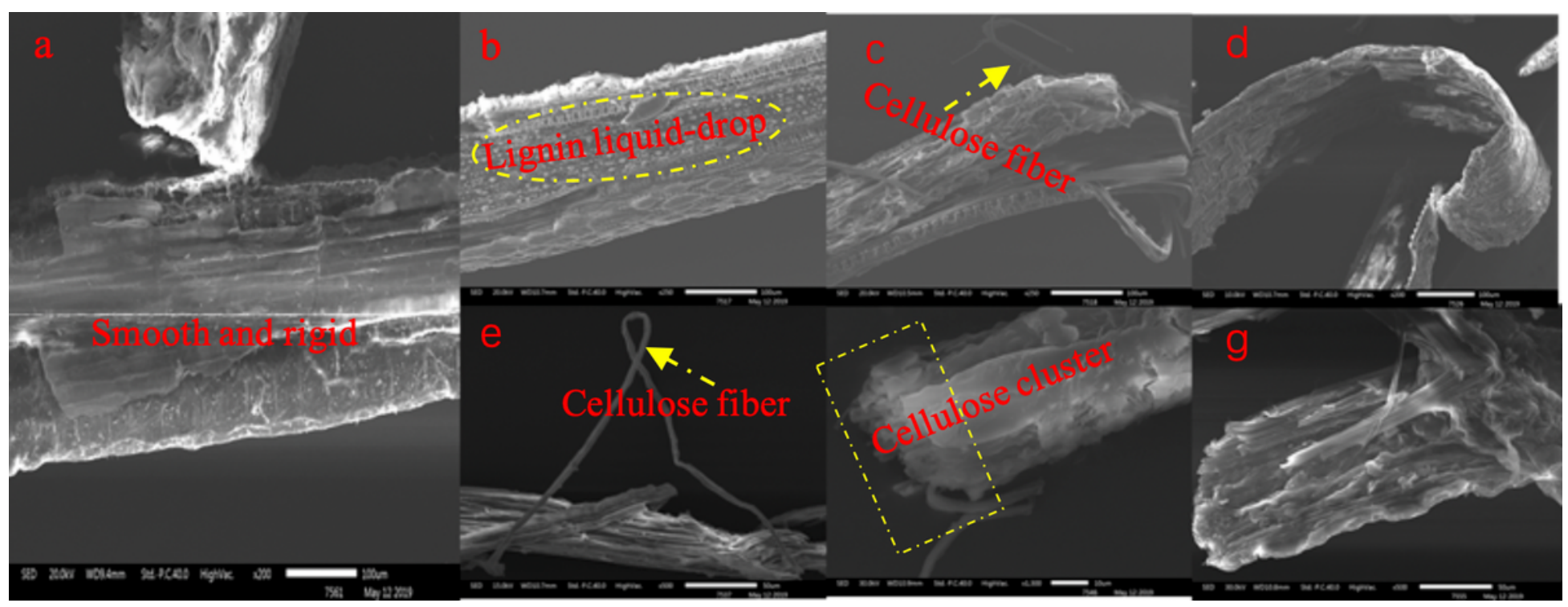

Figure 2

SEM of corn straw pretreatment under different condition (a: un-treatment, b: TH21, c: TH22, d: TH24, e: TH51, f: TH52, g: TH54)

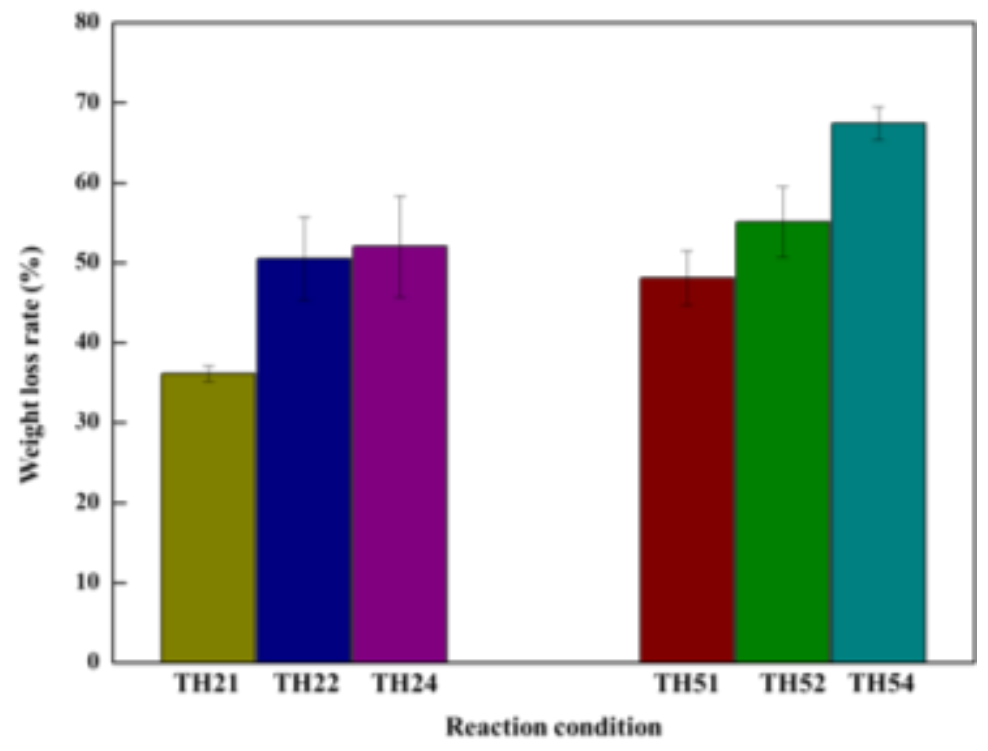

\section{Figure 3}

Weight loss of corn straw by the pretreatment under different conditions 


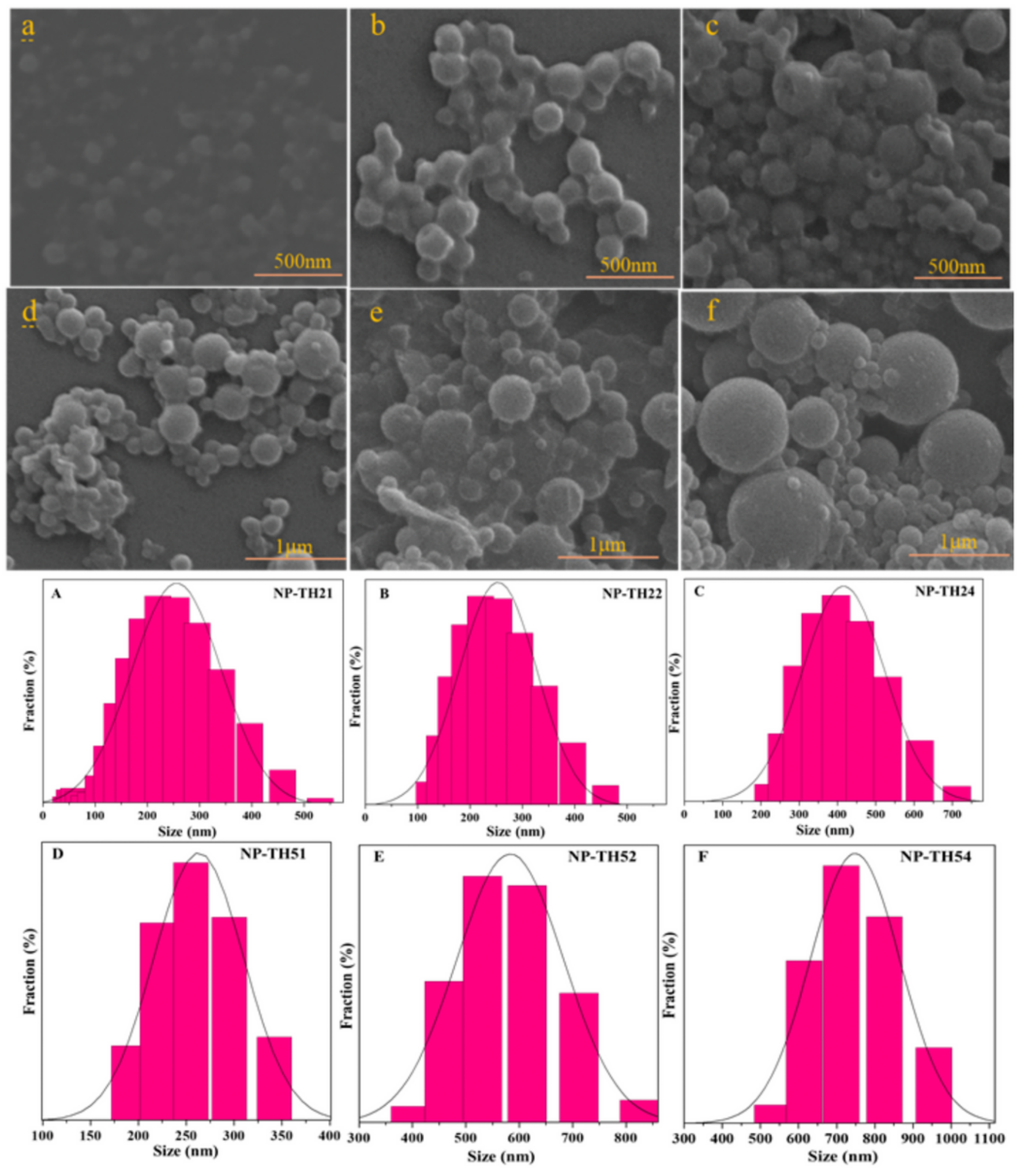

Figure 4

FESEM and Size Distribution of lignin nanoparticles prepared from different pretreatment liquid (a: NPTH21, b: NP-TH22, c: NP-TH24, d: NP-TH51, e: NP-TH52, f: NP-TH54) NP-THXX represents nanoparticles prepared from the pretreatment liquid under the condition of $X$ temperature $X$ hour. 

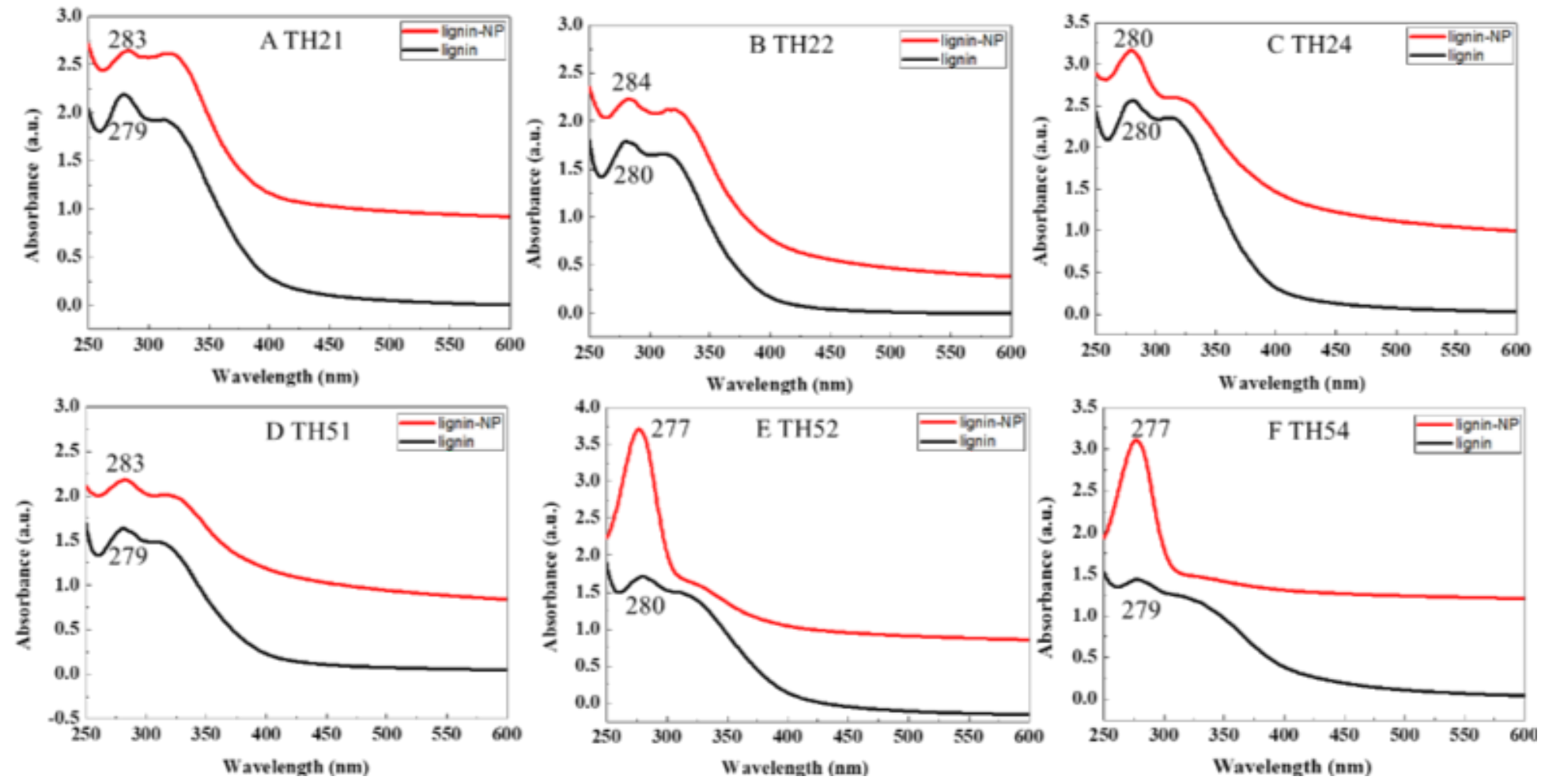

Figure 5

Uv-vis spectra of lignin and lignin nanoparticles prepared from different pretreatment liquid

A

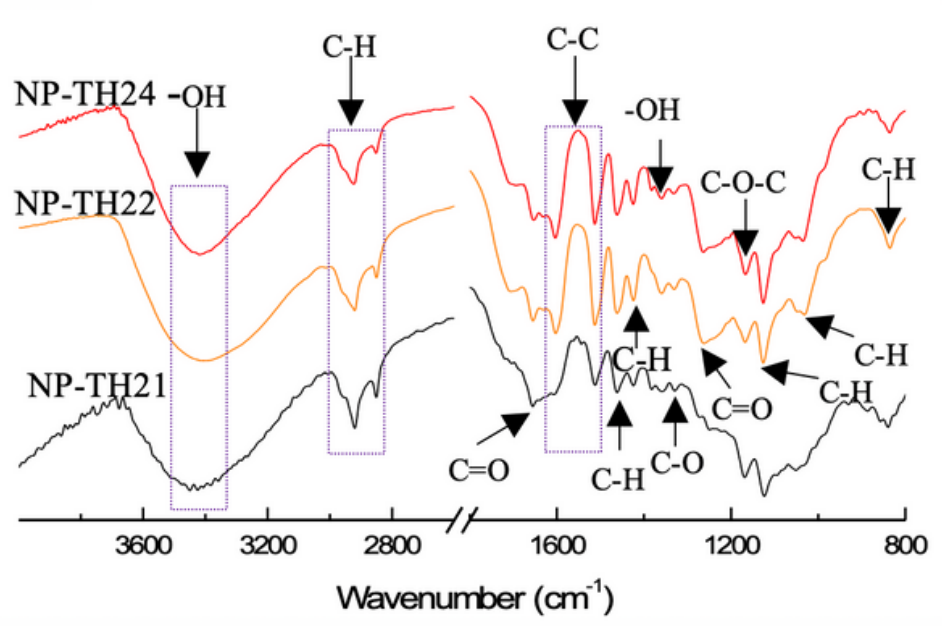

B

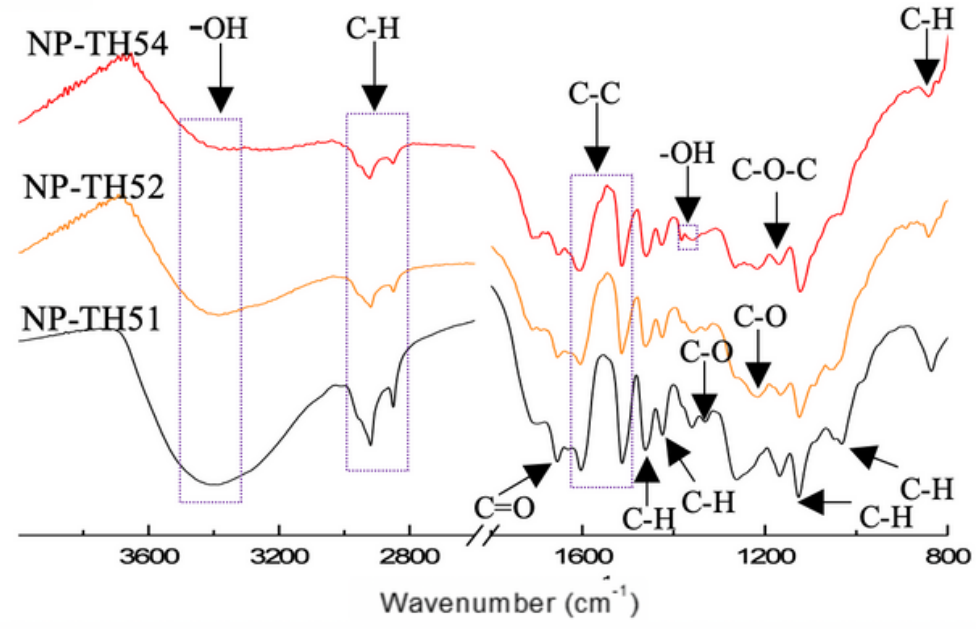

Figure 6

FTIR spectra of lignin nanoparticles prepared from different pretreatment liquid 


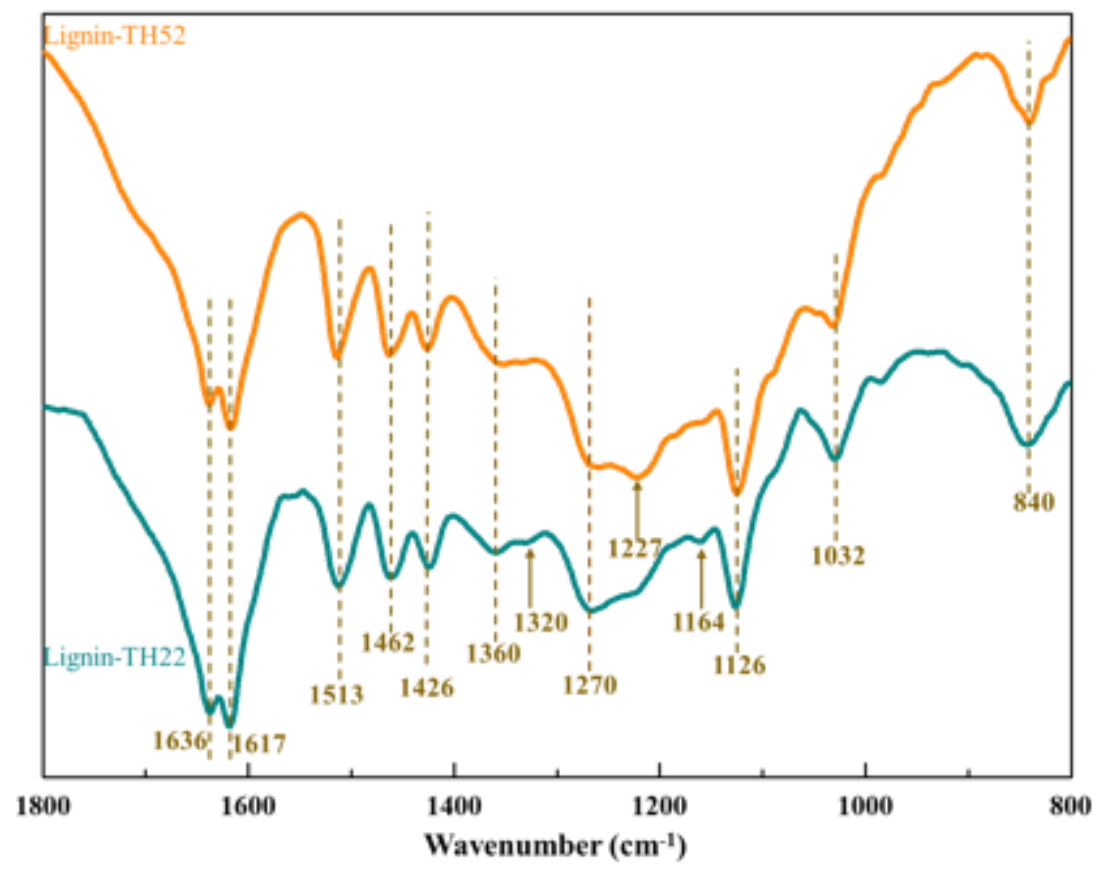

Figure 7

FTIR spectra of lignin extracted from $\mathrm{TH} 22$ and TH52
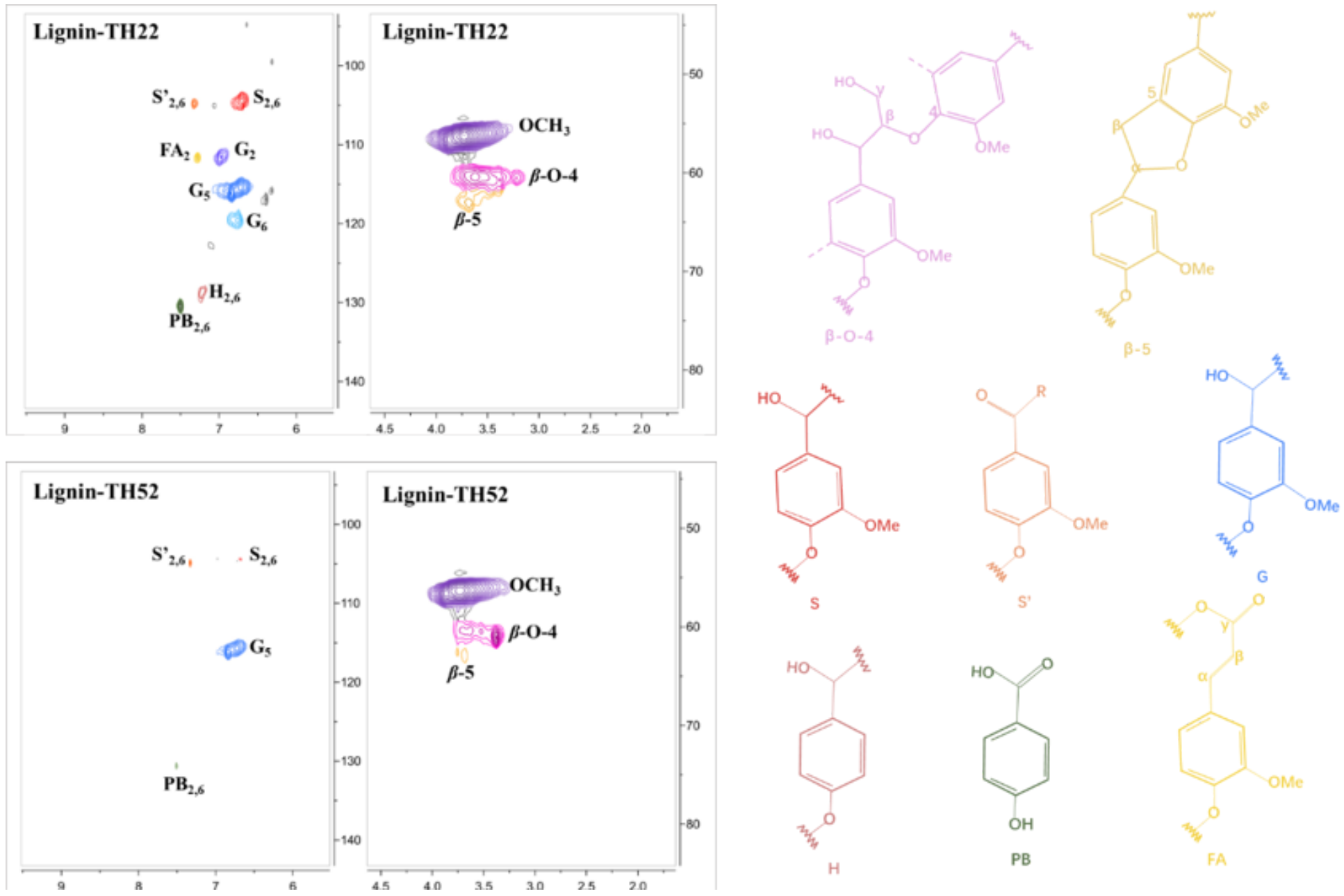

\section{Figure 8}


Aromatic region and side-chain in the HSQC-2DNMR spectra of lignins S: syringyl units, S': oxidized syringyl units, G: guaiacyl units, $\mathrm{H}$ : p-hydroxyphenyl units, $\mathrm{PB}$ : p-hydroxybenzoate substructures, FA: ferulates.

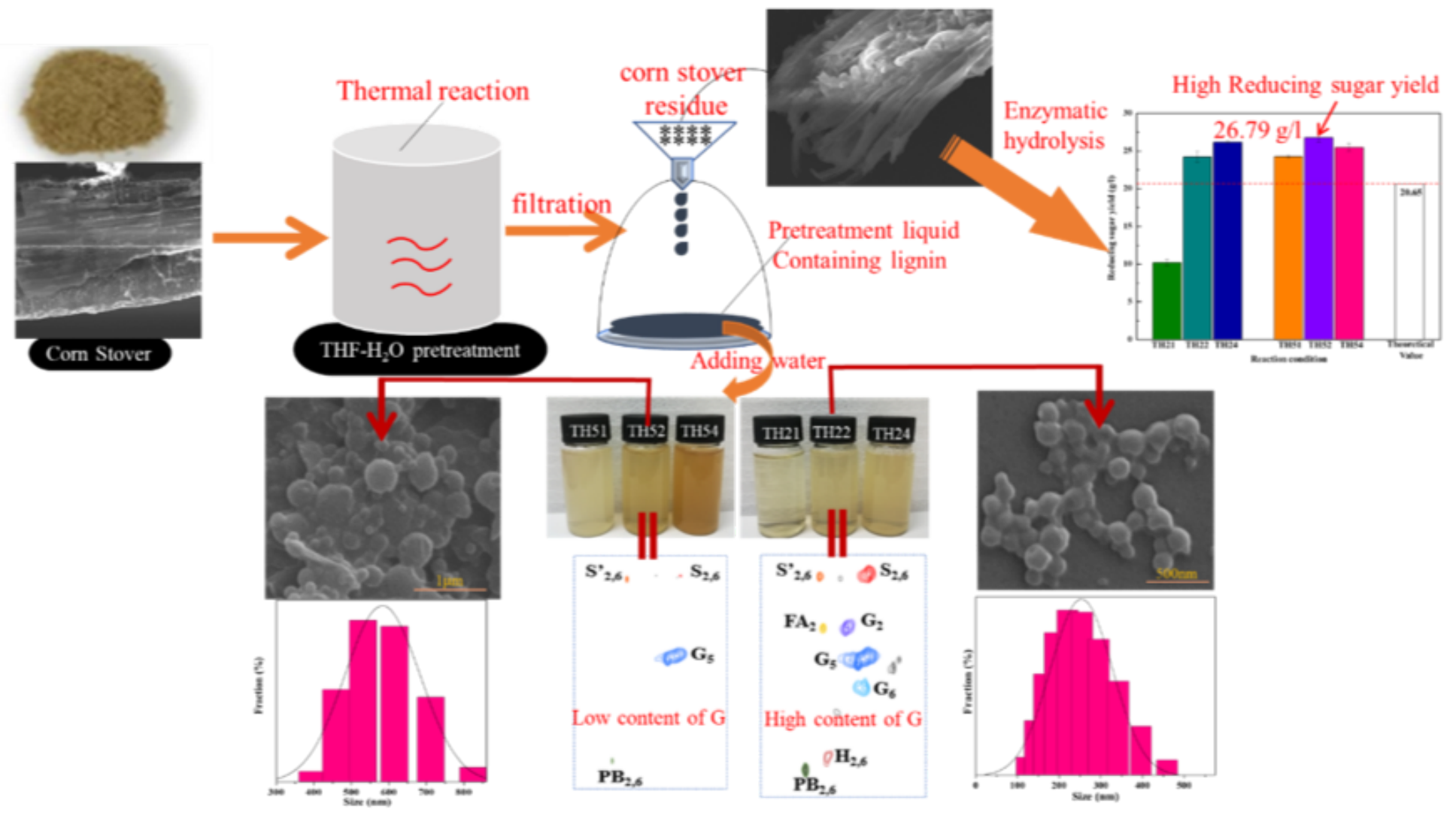

Figure 9

A schematic process flow diagram illustrating the high reducing sugar production and lignin nanoparticles formation from CS by THF-H2O pretreatment

\section{Supplementary Files}

This is a list of supplementary files associated with this preprint. Click to download.

- Additionalfile20191218.docx 